\title{
Advanced Practice Nurses Facilitating Clinical Translational Research
}

Lauren C. Schramp, BSN, RN; Maryann Holtcamp, MS, APN; Shane A. Phillips, PhD, PT; Timothy P. Johnson, PhD; and Julie Hoff, PhD, RN

Background: In 2006, the Clinical and Translational Science Award (CTSA), initiated by the National Center for Research Resources, was launched, with the overall goal to improve human health through clinical translational research. The purpose of this study was to examine the association between the total visit length for standardized study visits requiring the services of either a physician colleague or a designated advanced practice nurse (APN) for a protocol conducted in a clinical research center (CRC) supported with CTSA funds.

Methods: Data collected for administrative purposes between June 2008 and September 2009 were used. This retrospective study compared the total visit length for the standardized study visits of a single study protocol requiring a gluteal fat biopsy procedure performed by either a physician colleague (an MD in this case) or a CRC-based APN employed by the CRC.

Results: During the observed time period, 67 gluteal fat biopsy procedures were performed by one physician colleague, and 33 procedures were performed by one CRC-based APN. The mean total visit length of standardized study visits for research subjects treated by the physician colleague was $192.9 \pm 65.8$ minutes, whereas the mean total visit length of standardized study visits for research subjects treated by the APN was $154.8 \pm 40.9$ minutes $(P=.003)$. Generalized least squares random effects regression model further evaluated the association between provider and visit length while controlling for age, gender, percent body fat and physical activity. The type of provider $(P<.00 \mathrm{I})$ and gender $(P<.05)$ were associated with length of visit.

Conclusions: The introduction of a CRC-based APN to perform advanced procedures demonstrates an efficient use of resources to meet the advanced clinical needs of specific research protocols. In this retrospective study, the ready availability of an APN (employed by the CRC) to perform gluteal fat biopsies during standardized study visits led to a decrease in total visit length, and thus a more rapid "turnover" of clinical research space compared to the use of a physician colleague. To best meet the needs of clinical researchers, CRCs should consider the employment of other clinicians to complement the research facilitation services provided by registered nurses. The addition of an APN to the CRC research facilitation team is novel and lends itself to the spirit of the clinical translational research initiative; additional studies evaluating the contribution of other licensed practitioners to improve research facilitation efficiency are needed.

Keywords: Clinical nurse specialist; Clinical research; Nurse practitioner; Nursing research

Corresponding Author: Julie Hoff, RN, PhD, Assistant Professor; University of Illinois at Chicago; Department of Biobehavioral Health Science; $3840 \mathrm{~S}$. Wood St. (MC 596); Chicago, IL 606 I 2; Tel: 3 12-996-2937; Fax: 312-996-678I; Email: jahoff@uic.edu
Received: January 4, 2010

Revised: April 16, 2010

Accepted: April 28, 2010

doi: $10.3121 / \mathrm{cmr} .2010 .9 \mid 1$

Source of financial support: This project was supported by the University of Illinois at Chicago (UIC) Center for Clinical and Translational Science (CCTS), Award Number ULI RR029879 from the National Center for Research Resources. The content is solely the responsibility of the authors and does not necessarily represent the official views of the National Center for Research Resources or the National Institutes of Health. 
I

2006, the Clinical and Translational Science Award (CTSA), initiated by the National Center for Research Resources, was launched with the overall goal to improve human health. This award seeks to transform the research and training environment in order to eliminate the gap that currently exists between basic research and clinical application. It is anticipated that this initiative will improve human health by accelerating the application of clinical translational research findings from bench to bedside and bedside to community. ${ }^{1}$

Essential facilitators of any clinical research endeavor are the availability of clinical research nurses. The clinical acumen of these registered nurses is complemented by special training in the protection of human subjects while maintaining the integrity of a research protocol. They facilitate clinical research by working with consented healthy and ill adults and children. This role requires extensive clinical competence across the life span, research regulatory knowledge, and knowledge of clinical research methods and processes.

When a study protocol requires advanced physical assessment, clinical procedures, or diagnostic testing, research nurses traditionally rely on physician colleagues to assist in the completion of study visit procedures. Often times, physician colleagues struggle to balance research responsibilities with multiple clinical care responsibilities of inpatient rounds, outpatient clinic, or clinical procedures, as well as teaching commitments. In our experience, research subjects occasionally wait for extended periods of time for the physician colleague to arrive after being paged to the clinical research center (CRC) to complete the study visit procedures requiring advanced clinical skills. Extended waiting periods decrease efficiency and service satisfaction for the research subject and the $\mathrm{CRC}$ research facilitation team.

In outpatient settings, timeliness is a well-documented factor in client satisfaction. ${ }^{2}$ The addition of an advanced practice nurse (APN) to the CRC research facilitation team has the potential to enrich the available research services, improve research subject and investigator satisfaction, and utilize research resources more efficiently. Advanced practice nurses are registered nurses who hold at least a master's degree with specialization in a clinical area and possess in-depth clinical knowledge and skills, along with expertise in the assessment, diagnosis, and treatment of complex health problems. ${ }^{3}$ Compared to other nurses in the CRC, APNs have advanced training and can be credentialed to deliver medical services once reserved only for physicians. Through collaborative agreements and clinical privileging, an APN may perform advanced physical assessments, clinical procedures, or diagnostic testing required by a study protocol. Their presence also makes advanced skills available to the clinical research nurse should an adverse event occur. While these occurrences are limited, having an APN on staff at a CRC can be beneficial in the event of an adverse clinical finding that requires immediate medical decision-making and management, and may even operate in a proactive preventative manner to avert such occurrences.

The addition of an APN to the CRC research facilitation team is novel and lends itself to the spirit of the clinical translational research initiative. This paper describes one six-month outcome of adding a CRC-based APN to the research facilitation team for a single study protocol.

\section{Methods}

This study was performed at a CTSA recipient university located in the Midwestern United States. The CRC, one component of the CTSA award, facilitates the research of more than 40 clinical investigators. This study compared the total visit length of standardized study visits for a protocol investigating vascular health before and after exercise in healthy subjects. Study subjects were asked to complete two study visits approximately two weeks apart, which included cardiovascular screening, vascular ultrasound, a short exercise session, anthropometric measures, phlebotomy, and subcutaneous fat biopsies to determine microvascular function.

The total visit length was defined as the elapsed time between the subject's arrival to and departure from the CRC. It was not the intention of this study to examine skill differences between providers; rather, the investigators sought to determine whether the addition of a CRC-based APN (a clinical nurse specialist) credentialed and privileged to perform minor surgical procedures (ie, gluteal fat biopsies) altered the total visit length compared to paging a physician colleague when a clinical research protocol required advanced clinical skills.

The primary protocol, from where the visit data originated, investigated vascular health before and after exercise in healthy subjects. The first standardized study visit included a brief medical history, collection of blood and urine specimens, a Dual Energy X-ray Absorptiometry (DEXA) scan, and a subcutaneous fat biopsy. The second standardized study visit included collection of blood and urine specimens, a brachial artery ultrasound, an exercise session, and a subcutaneous fat biopsy. As is apparent from the list of procedures, the fat biopsy is the only procedure which requires specially licensed and credentialed health care personnel. Subcutaneous fat biopsy credentialing of the APN was established through direct observation and training with the CRC collaborating surgeon.

Visits to the CRC were recorded in an electronic database. Age, gender, activity status (runner, weight-lifter, both, or inactive), and percent body fat were collected at the first visit, along with the arrival time and departure time. On the second visit, which usually occurred within 14 days, only arrival time and departure time were collected. The physician colleague and APN were informed of all scheduled study visits at least 
Table 1. Sample characteristics by provider and gender.

\begin{tabular}{|c|c|c|c|c|c|c|c|}
\hline \multirow[b]{2}{*}{ Characteristic } & \multicolumn{2}{|c|}{ Total } & \multirow[b]{2}{*}{$\boldsymbol{P}$} & \multicolumn{2}{|c|}{ Physician } & \multicolumn{2}{|c|}{ APN } \\
\hline & Men & Women & & Men & Women & Men & Women \\
\hline $\mathrm{N}$ & 29 & 24 & & 16 & 19 & 13 & 5 \\
\hline Age $(\mathrm{y})$, mean (SD) & $\begin{array}{l}26.66 \\
(5.11)\end{array}$ & $\begin{array}{l}27.29 \\
(5.74)\end{array}$ & NS & $\begin{array}{l}26.2 \\
(5.0)\end{array}$ & $\begin{array}{l}27.3 \\
(6.1)\end{array}$ & $\begin{array}{l}27.2 \\
(5.3)\end{array}$ & $\begin{array}{l}27.4 \\
(4.6)\end{array}$ \\
\hline Body fat (\%), mean (SD) & $\begin{array}{l}16.64 \\
(6.36)\end{array}$ & $\begin{array}{l}27.52 \\
(7.28)\end{array}$ & $<.001$ & $\begin{array}{l}18.7 \\
(7.3)\end{array}$ & $\begin{array}{l}26.9 \\
(7.6)\end{array}$ & $\begin{array}{l}13.8 \\
(3.5)\end{array}$ & $\begin{array}{l}29.7 \\
(6.0)\end{array}$ \\
\hline Visit length (min), mean (SD) & $\begin{array}{l}153.4 \\
(57.0)\end{array}$ & $\begin{array}{l}177.4 \\
(48.0)\end{array}$ & NS & $\begin{array}{l}172.8 \\
(66.3)\end{array}$ & $\begin{array}{l}176.6 \\
(47.2)\end{array}$ & $\begin{array}{l}129.6 \\
(30.7)\end{array}$ & $\begin{array}{c}180 \\
(56.7)\end{array}$ \\
\hline
\end{tabular}

24 hours in advance. Data were examined using descriptive and inferential statistics. Statistical analyses were performed using SPSS (SPSS for Windows, Release 16.0.1, 2007. Chicago, IL: SPSS Inc. 2007) and Stata Statistical Software (Stata Statistical Software, Release 10. College Station, TX: StataCorp LP. 2007). Institutional review board approval was granted for this study.

\section{Results}

Between June 2008 and September 2009, 47 healthy subjects underwent two gluteal fat biopsy procedures each, and six underwent only a single gluteal fat biopsy procedure (due to dropout) in the CRC. The sample consisted of 24 women and 29 men, whose baseline mean age did not differ, while the body fat percentage was significantly greater in women $(t=5.76, d f=50, P<.001)$. The activity status of a majority of subjects, $90 \%$ men and 54\% women, was self-reported as "active." The gluteal fat biopsy procedure was performed on 67 occasions by the physician colleague and 33 occasions by the APN. Table 1 summarizes additional sample characteristics by provider and gender.

Analysis by $t$ test revealed that the mean visit time for study subjects treated by a physician colleague was $192.9 \pm 65.84$ minutes, whereas the mean visit time for study subjects treated by the APN was $154.85 \pm 40.9$ minutes. This observed mean difference of almost 40 minutes in study visit time was statistically significant $(t=3.55, d f=93, P=.003)$. All biopsies were performed successfully and without complication, and the other study procedures (cardiovascular screening, vascular ultrasound, a short exercise session, anthropometric measures, and phlebotomy) were performed according to a standardized protocol. For the physician colleague, visit times ranged from 40 minutes to 380 minutes, while for the APN, times ranged more narrowly from 85 minutes to 280 minutes. This observed study visit time difference was associated with the amount of time waiting for the physician colleague to arrive at the CRC to perform the biopsy.

To further examine the association between the total visit length for standardized study visits requiring the services of either a physician colleague or a CRC-based APN, a multivariate regression model was next examined. Due to the fact that multiple observations were included for some patients, a generalized least squares random effects regression model was estimated, rather than an ordinary least squares model. Normality assumptions were met for all variables included in this model. Results are presented in table 2 . Differences in length of visit by type of provider continued to be significant after controlling for patient age, gender, percent body fat, and physical activity. Of these control variables, only gender was found to also be independently associated with length of visit. Specifically, study visits took longer for women than for men. Overall, the model accounted for about $15 \%$ of the variability in length of the health care visits examined $\left(\mathrm{R}^{2}=0.154\right)$.

\section{Discussion}

Efficient utilization of resources is vital to the success of a CRC. Numerous studies report the effectiveness of APN services in clinical settings. ${ }^{4-6}$

Introduction of an APN to the CRC led to more rapid "turnover" of clinical research space and decreased the length of nursing support time required for each standardized study visit. A CRC-based APN was present from the start of the study visit, and the research subject was moved through each study visit with minimal waiting time between study procedures. Unlike the physician colleague, the APN was also available throughout the study visit to address any questions regarding the biopsy and to review and reinforce biopsy site care before and after the procedure.

This study does have limitations. It was a retrospective analysis of data collected for administrative purposes. Precise time measurements were not recorded for each study visit procedure. The study sample consisted of a convenience sample of healthy, self-selected individuals who agreed to participate in a research protocol examining vascular health before and after exercise. Cost-effectiveness comparing the APN to the physician colleague was not part of the study design.

In the multivariate analysis, study visits were longer for women compared to men. While the reason for the gender difference in total study visit length is not clear, it is possible 
Table 2. Random effects GLS regression of length of visit.

\begin{tabular}{lcccc}
\hline Characteristic & Coefficient & SE & z & $\boldsymbol{P}$ \\
\hline Provider type & -31.97 & -15.19 & -2.1 & 0.035 \\
Patient age, y & -1.91 & -1.35 & -1.41 & 0.157 \\
Patient gender & -38.07 & -18.8 & -2.02 & 0.043 \\
Body fat, \% & -1.04 & -1.05 & -0.98 & 0.326 \\
Activity status & 13.29 & -17.63 & 0.75 & 0.451 \\
Constant & 307.2 & -51.66 & 5.95 & 0.000 \\
\hline
\end{tabular}

GLS, generalized least squares

that the known differences in body fat distribution patterns and gluteal fat cell characteristics between healthy men and women may influence the length of the biopsy procedure. During a gluteal fat biopsy, differences in gluteal fat cell size and distribution are visibly apparent. ${ }^{7,8}$ Fat cells in women appear to be more diffuse; therefore, additional minutes may be required to obtain a gluteal fat biopsy specimen. The role of fat distribution on procedure time requires further study.

\section{Conclusion}

As CRCs evolve through clinical translational research funding, creative ways to meet the needs of research subjects and investigators must be explored to improve efficiency and research facilitation service satisfaction. The introduction of an APN to a research facilitation team assists with this goal and complements the mission of a CRC while also bringing a higher level of nursing practice to the research unit.

To the best of our knowledge, we are the first to present any data specific to this subject and setting. Though we believe other CRCs are utilizing APNs, we were unable to find in the literature any data on their effectiveness in this setting. Future research should include more precise measurements of study procedure phases and measurements of research subject and investigator satisfaction. We report an association between the total visit length for standardized study visits requiring the services (gluteal fat biopsy) of either a physician colleague or a designated APN for a protocol conducted in a CRC. Further studies are needed to ascertain why such differences exist and how staff make-up can affect CRC performance. As additional services are added to a CRC, prospective evaluation studies are needed to assess clinical and cost-effectiveness.

\section{Acknowledgments}

The authors wish to acknowledge the computer programming assistance of Dr. Young Ik Cho and the editorial assistance of Mr. Kevin Grandfield, MFA.

\section{References}

1. Clinical and Translational Science Awards: Advancing scientific discoveries nationwide to improve health. Progress report 2006-2008. NIH Publication No. 09-7404; National Center for Research Resources, 2009. 11-12.

2. Leddy KM, Kaldenberg DO, Becker BW. Timeliness in ambulatory care treatment. An examination of patient satisfaction and wait time in medical practices and outpatient test and treatment facilities. J Ambul Care Manage 2003;26:138-149.
3. Consensus Model for APRN Regulation: Licensure, Accreditation, Certification \& Education. APRN Consensus Work Group. 2008. Available at: http://www.aacn.nche.edu/ Education/pdf/APRNReport.pdf.

4. American Academy of Nurse Practitioners. Nurse Practitioner Cost-Effectiveness. Office of Health Policy. 2007. Available at: http://www.aanp.org/NR/rdonlyres/197C9C42-4BC142A5-911E-85FA759B0308/0/CostEffectiveness4pages.pdf.

5. Gambino KG, Planavsky L, Gaudette H. Transition toward a nurse practitioner managed clinic. J Cardiovasc Nurs 2009;24:132-139.

6. Klaasen K, Lamont L, Krishnan P. Setting a new standard of care in nursing homes. Can Nurse 2009; 105:24-30.

7. Votruba SB, Jensen MD. Sex differences in abdominal, gluteal, and thigh LPL activity. Am J Physiol Endocrinol Metab 2007;292:E1523-E1528.

8. Lawlor DA, Ebrahim S, Whincup P, Sterne J, Papacosta O, Wannamethee G, Dhanjil S, Griffin M, Nicolaides AN, Davey Smith G. Sex differences in body fat distribution and carotid intima media thickness: cross sectional survey using data from the British regional heart study. J Epidemiol Community Health 2004;58:700-704.

\section{Author Affiliations}

Lauren C. Schramp, BSN, $R N{ }^{*}{ }^{*}$;

Maryann Holtcamp, MS, APN';

Shane A. Phillips, PhD, PTt;

Timothy P. Johnson, PhD广;

Julie Hoff, $P h D, R N^{* \dagger}$

*University of Illinois at Chicago, College of Nursing,

Chicago, IL USA

†University of Illinois Center for Clinical and Translational

Science, Chicago, IL USA

FUniversity of Illinois at Chicago, College of Applied Health

Science, Dept. of Physical Therapy, Chicago, IL USA 